\title{
Controllability of nonlinear neutral fractional impulsive differential inclusions in Banach space
}

Yong $L i^{*}$

\section{"Correspondence:}

yongli260@163.com

Institute of Mathematics Science,

Chongqing Normal University,

Chongqing, 400047, P.R. China

\section{黛 Springer}

\begin{abstract}
The paper is concerned with the controllability of nonlinear neutral fractional impulsive differential inclusions with infinite delay in a Banach space. Sufficient conditions for the controllability are obtained by using a fixed point theorem due to Dhage.

Keywords: mild solution; convex multivalued map; neutral impulsive differential inclusions; controllability; infinite delay
\end{abstract}

\section{Introduction}

Fractional differential equations have been proved to be one of the most effective tools in the modeling of many phenomena in various fields of physics, mechanics, chemistry, engineering, etc. For more details, see [1-5]. In order to describe various real-world problems in physical and engineering science subject to abrupt changes at certain instants during the evolution process, impulsive differential equations have been used to model the systems. The theory of impulsive differential equations is an important branch of differential equations, which has an extensive physical background [6-8].

Controllability is one of the important fundamental concepts in mathematical control theory and plays an important role in control systems. The problem of controllability is to show the existence of a control function, which steers the solution of the system from its initial state to a final state, where the initial and final states may vary over the entire space. A standard approach is to transform the controllability problem into a fixed point problem for an appropriate operator in a functional space. The problem of controllability and optimal controls for functional differential systems have been extensively studied in many papers [9-23]. For example, Wang JinRong and Zhou Yong [9] proved the existence and controllability results for fractional semilinear differential inclusions involving the Caputo derivative in Banach spaces by using operator semigroups and Bohnenblust-Karlin's fixed point theorem. Wang Jinrong et al. [11] established two sufficient conditions for nonlocal controllability for fractional evolution systems under some weak compactness conditions. Wang Jinrong et al. [13] considered the nonlinear control systems of fractional order and its optimal controls in Banach spaces and the sufficient condition is given for the existence and uniqueness of mild solutions for a broad class of fractional nonlinear infinite dimensional control systems. Wang Jinrong et al. [14] studied optimal feedback controls of a

2014 Li; licensee Springer. This is an Open Access article distributed under the terms of the Creative Commons Attribution License (http://creativecommons.org/licenses/by/2.0), which permits unrestricted use, distribution, and reproduction in any medium, provided the original work is properly cited. 
system governed by semilinear fractional evolution equations via a compact semigroup in Banach spaces. Wang Jinrong et al. [15] studied optimal relaxed controls and relaxation of nonlinear fractional impulsive evolution equations. Wang Jinrong et al. [16] investigated a class of Sobolev type semilinear fractional evolution systems in a separable Banach space. Applying a suitable fixed point theorem as well as condensing mapping, controllability results for two classes of control sets are established by means of the theory of propagation family and the technique of the measure of noncompactness. In [17], Chang YongKui et al. established a sufficient condition for the controllability of impulsive neutral functional differential inclusions in Banach space by using the Dhage fixed point theorem. M Benchohra et al. [24] discussed the controllability for first-order, second-order functional differential and integrodifferential inclusions in Banach space with finite delay. Jong Yeoul Park et al. [25] discussed the controllability for second-order neutral functional differential inclusions in Banach space with the help of some fixed point theorems. In [26, 27], Bing Liu investigated the controllability of neutral functional differential and integrodifferential inclusions with infinite delay. P Balasubramaniam and SK Ntouyas [28] obtained the controllability result of stochastic differential inclusions with infinite delay in abstract space. R Sakthivel et al. [18] considered a class of fractional neutral control systems governed by abstract nonlinear fractional neutral differential equations and established a new set of sufficient conditions for the controllability of nonlinear fractional systems by using a fixed point analysis approach. Using fixed point techniques, fractional calculations, stochastic analysis techniques and methods adopted directly from deterministic control problems, R Sakthivel et al. [20] gave a new set of sufficient conditions for approximate controllability of fractional stochastic differential equations. In [21], R Sakthivel and Y Ren investigated the complete controllability property of a nonlinear stochastic control system with jumps in a separable Hilbert space.

Since many systems arising from realistic models heavily depend on histories (i.e., there is the effect of infinite delay on the state equations) [31], there is a real need to discuss partial functional differential systems with infinite delay. So, in the present paper, we will concentrate on the case with infinite delay and establish sufficient conditions for the controllability of systems (1.1) by relying on a fixed point theorem due to Dhage [29].

In this paper we will concentrate on the case with infinite delay and impulsive effect, and establish sufficient conditions for the controllability of the following fractional impulsive differential inclusions:

$$
\left\{\begin{array}{c}
{ }^{C} D_{t}^{\alpha}\left[x(t)-g\left(t, x_{t}\right)\right] \in A x(t)+F\left(t, x_{t}\right)+(B u)(t), \\
t \in J=[0, b], t \neq t_{k}, k=1,2, \ldots, m, \\
\left.\triangle x\right|_{t=t_{k}}=I_{k}\left(x\left(t_{k}^{-}\right)\right), \quad k=1,2, \ldots, m, \\
x_{0}=\phi \in \mathfrak{B}_{h}, \quad t \in J_{0}=(-\infty, 0],
\end{array}\right.
$$

where ${ }^{C} D_{t}^{\alpha}$ is the Caputo fractional derivative of order $0<\alpha<1$; the state $x(\cdot)$ takes values in Banach space $X$ with the norm $|\cdot|, A$ is the infinitesimal generator of an analytic semigroup of the bounded linear operator $\{T(t), t \geq 0\}$ in $X$, the control function $u(\cdot)$ is given in $L^{2}(J, U)$, and we have a Banach space of admissible control functions with $U$ as a Banach space. $F: J \times \mathfrak{B}_{h} \rightarrow \mathcal{P}(X)$ is a bounded, closed, convex-valued multivalued map, $g: J \times \mathfrak{B}_{h} \rightarrow X$ are given functions, where $\mathfrak{B}_{h}$ is a phase space defined in preliminaries. $0=t_{0}<t_{1}<\cdots<t_{m}<t_{m+1}=b, I_{k} \in C(X, X)(k=1,2, \ldots, m)$ are bound functions. 
$\left.\triangle x\right|_{t=t_{k}}=x\left(t_{k}^{+}\right)-x\left(t_{k}^{-}\right), x\left(t_{k}^{-}\right), x\left(t_{k}^{+}\right)$represent the left and right limit of $x(t)$ at $t=t_{k} \cdot \mathcal{P}(X)$ denotes the class of all nonempty subsets of $X$. The histories $x_{t}:(-\infty, 0] \rightarrow X, x_{t}(s)=x(t+s)$, $s \leq 0$, belong to an abstract phase space $\mathfrak{B}_{h}$.

The structure of this paper is as follows. In Section 2 we briefly present some basic notations and preliminaries. The controllability result of system (1.1) is investigated by means of a fixed point theorem and operator theory in Section 3. A conclusion is given in Section 4 .

\section{Preliminaries}

Let $(E,\|\cdot\|)$ be a Banach space. A multivalued map $\mathfrak{J}: E \rightarrow 2^{E}$ is convex (closed)-valued, if $\mathfrak{J}(x)$ is convex (closed) for all $x \in E$. $\mathfrak{J}$ is bounded on a bounded set if $\mathfrak{J}(B)=\bigcup_{x \in B} \mathfrak{J}(x)$ is bounded in $E$ for any bounded set $B$ of $E$; i.e.,

$$
\sup _{x \in B} \sup \{\|y\| \in \mathfrak{J}(x)\}<\infty .
$$

$\mathfrak{J}$ is called upper semicontinuous (u.s.c.) on $E$, if for each $x_{*} \in E$, the set $\mathfrak{J}\left(x_{*}\right)$ is a nonempty, closed subset of $E$, and if for each open set $B$ of $E$ containing $\mathfrak{J}\left(x_{*}\right)$, there exists an open neighborhood $V$ of $x_{*}$ such that $\mathfrak{J}(V) \subseteq B$.

$\mathfrak{J}$ is said to be completely continuous if $\mathfrak{J}(B)$ is relatively compact, for every bounded subset $B \subseteq E$.

If the multivalued map $\mathfrak{J}$ is completely continuous with nonempty compact values, then $\mathfrak{J}$ is u.s.c. if and only if $\mathfrak{J}$ has a closed graph (i.e., $x_{n}=x_{*}, y_{n}=y_{*}, y_{n} \in \mathfrak{J} x_{n}$ imply $y_{*} \in \mathfrak{J} x_{*}$ ).

Let $B C C(E)$ denote the set of all the set of all nonempty, bounded, closed, and convex subsets of $E$. For more details of multivalued maps see the books of Deimling [32], and of $\mathrm{Hu}$ and Papageorgiou [33].

If $T$ is an uniformly bounded and analytic semigroup with infinitesimal generator $A$ such that $0 \in \rho(A)$ then it is possible to define the fractional power $(-A)^{\alpha}$, for $0<\alpha \leq 1$, as a closed linear operator on its domain $D\left((-A)^{\alpha}\right)$. Furthermore, the subspace $D\left((-A)^{\alpha}\right)$ is dense in $X$ and the expression

$$
\|x\|_{\alpha}:=\left\|(-A)^{\alpha} x\right\|, \quad x \in D\left((-A)^{\alpha}\right)
$$

defines a norm on $D\left((-A)^{\alpha}\right)$. Hereafter we represent by $X_{\alpha}$ the space $D\left((-A)^{\alpha}\right)$ endowed with the norm $\|\cdot\|_{\alpha}$. We suppose that $A$ is the infinitesimal generator of an analytic semigroup of bounded linear operators $T(t), 0 \in \rho(A)$, for $t \geq 0$, then there exist constants $M$ such that $|T(t)| \leq M$. Then the following properties are well known [34].

Lemma 2.1 [34] Suppose that the preceding conditions are satisfied.

(a) Let $0<\alpha \leq 1$. Then $X_{\alpha}$ is a Banach space.

(b) If $0<\gamma<\alpha \leq 1$ then $X_{\alpha} \hookrightarrow X_{\gamma}$ and the embedding is compact whenever the resolvent operator of $A$ is compact.

(c) For every $a>0$, there exists a positive constant $c_{\alpha}$ such that

$$
\left\|(-A)^{\alpha} T(t)\right\| \leq \frac{c_{\alpha}}{t^{\alpha}}, \quad 0<t \leq a .
$$


Definition 2.1 The fractional integral of order $\alpha$ with the lower limit 0 for a function $f$ is defined as

$$
I^{\alpha} f=\frac{1}{\Gamma(\alpha)} \int_{0}^{t} \frac{f(s)}{(t-s)^{1-\alpha}} d s, \quad t>0, \alpha>0,
$$

provided the right-hand side is pointwise defined on $[0, \infty)$. Here $\Gamma(\cdot)$ is the gamma function.

Definition 2.2 The Caputo derivative of order $\alpha$ with the lower limit 0 for a function $f$ can be written as

$$
{ }^{C} D^{\alpha} f(t)=\frac{1}{\Gamma(n-\alpha)} \int_{0}^{t} \frac{f^{n}(s)}{(t-s)^{\alpha+1-n}} d s=I^{n-\alpha} f^{n}(t), \quad t>0,0 \leq n-1<\alpha<n .
$$

The key tool in our approach is the following fixed point theorem [24].

Theorem 2.2 ([29] Dhage's fixed point theorem) Let $X$ be a Banach space. $\Phi_{1}: X \rightarrow$ $\mathcal{P}_{c l, c v, b d}(X)$ and $\Phi_{2}: X \rightarrow \mathcal{P}_{c p, c v}(X)$ be two multivalued operators satisfying:

(a) $\Phi_{1}$ is a contraction, and

(b) $\Phi_{2}$ is completely continuous.

Then either:

(i) the operator inclusion $\lambda x \in \Phi_{1} x+\Phi_{2} x$ has a solution for $\lambda=1$, or

(ii) the set $G=\left\{x \in X: \lambda x \in \Phi_{1} x+\Phi_{2} x, \lambda>1\right\}$ is unbounded.

We present the abstract phase space $\mathfrak{B}_{h}$, which has been used in [17, 27]. Assume that $h:(-\infty, 0] \rightarrow(0,+\infty)$ is a continuous function with $l=\int_{-\infty}^{0} h(t) d t\langle+\infty$. For any $a\rangle$ 0 , we define $\mathfrak{B}=\{\psi:[-a, 0] \rightarrow X$ such that $\psi(t)$ is bounded and measurable $\}$ and equip the space $\mathfrak{B}$ with the norm $\|\psi\|_{[-a, 0]}=\sup _{s \in[-a, 0]}|\psi(s)|, \forall \psi \in \mathfrak{B}$. Let us define $\mathfrak{B}_{h}=\{\psi$ : $(-\infty, 0] \rightarrow X$ such that, for any $c>0,\left.\psi\right|_{[-c, 0]} \in \mathfrak{B}$ and $\left.\int_{-\infty}^{0} h(s)\|\psi\|_{[s, 0]} d s<+\infty\right\}$.

If $\mathfrak{B}_{h}$ is endowed with the norm $\|\psi\|_{\mathfrak{B}_{h}}=\int_{-\infty}^{0} h(s)\|\psi\|_{[s, 0]} d s, \forall \psi \in \mathfrak{B}$, then it is clear that $\left(\mathfrak{B}_{h},\|\cdot\|_{\mathfrak{B}_{h}}\right)$ is a Banach space $[17,27]$. Now we consider the space $\mathfrak{B}_{b}=\{\psi:(-\infty, b] \rightarrow$ $X$ such that $x_{k} \in C\left(J_{k}, X\right)$ and there exist $x\left(t_{k}^{+}\right)$and $x\left(t_{k}^{-}\right)$with $x\left(t_{k}\right)=x\left(t_{k}^{-}\right), x_{0}=\phi \in \mathfrak{B}_{h}, k=$ $0,1,2, \ldots, m\}$ where $x_{k}$ is the restriction of $x$ to $J_{k}=\left(t_{k}, t_{k+1}\right], k=0,1,2, \ldots, m$. Set $|\cdot|_{b}$ be a seminorm in $\mathfrak{B}_{b}$ defined by

$$
\|x\|_{b}=\left\|x_{0}\right\|_{\mathfrak{B}_{h}}+\sup \{|x(s)|: s \in[0, b]\}, \quad x \in \mathfrak{B}_{b}
$$

\section{Main result}

In the following, we shall apply Theorem 2.2 to the study of the controllability of system (1.1).

Definition 3.1 A function $x:(-\infty, b] \rightarrow X$ is called a mild solution of system (1.1) if the following holds: $x_{0}=\phi \in \mathfrak{B}_{h}$ on $(-\infty, 0],\left.\Delta x\right|_{t=t_{k}}=I_{k}\left(x\left(t_{k}^{-}\right)\right), k=1,2, \ldots, m$, the restriction 
of $x(\cdot)$ to the interval $[0, b)-\left\{t_{1}, t_{2}, \ldots, t_{m}\right\}$ is continuous and the integral equation

$$
\left\{\begin{aligned}
x(t)= & S_{\alpha}(t)[\phi(0)-g(0, \phi)]+g\left(t, x_{t}\right)+\int_{0}^{t}(t-s)^{\alpha-1} A T_{\alpha}(t-s) g\left(s, x_{s}\right) d s \\
& +\int_{0}^{t}(t-s)^{\alpha-1} T_{\alpha}(t-s) f(s) d s+\int_{0}^{t}(t-s)^{\alpha-1} T_{\alpha}(t-s)(B u)(s) d s \\
& +\sum_{0<t_{k}<t} S_{\alpha}\left(t-t_{k}\right) I_{k}\left(x\left(t_{k}^{-}\right)\right), \quad t \in J, \\
x_{0}=\phi & \in \mathfrak{B}_{h}, \quad t \in J_{0},
\end{aligned}\right.
$$

is satisfied, where

$$
\begin{aligned}
& f \in S_{F, x}=\left\{f \in L^{1}(J, X): f(t) \in F\left(t, x_{t}\right), \text { for a.e. } t \in J\right\}, \\
& S_{\alpha}(t)=\int_{0}^{\infty} \xi_{\alpha}(\theta) T\left(t^{\alpha} \theta\right) d \theta, \quad T_{\alpha}(t)=\alpha \int_{0}^{\infty} \theta \xi_{\alpha}(\theta) T\left(t^{\alpha} \theta\right) d \theta, \\
& \xi_{\alpha}(\theta)=\frac{1}{\alpha} \theta^{-1-\frac{1}{\alpha}} \varpi_{\alpha}\left(\theta^{-\frac{1}{\alpha}}\right) \geq 0, \\
& \varpi_{\alpha}(\theta)=\frac{1}{\pi} \sum_{n=1}^{\infty}(-1)^{n-1} \theta^{-n \alpha-1} \frac{\Gamma(n \alpha+1)}{n !} \sin (n \pi \alpha), \quad \theta \in(0, \infty) .
\end{aligned}
$$

$\xi_{\alpha}$ is a probability density function defined on $(0, \infty)$, that is, $\xi_{\alpha}(\theta) \geq 0, \theta \in(0, \infty)$, and $\int_{0}^{\infty} \xi_{\alpha}(\theta) d \theta=1$.

Lemma 3.1 [30] The operators $S_{\alpha}(t)$ and $T_{\alpha}(t)$ have the following properties:

(1) For any fixed $t>0, S_{\alpha}(t)$ and $T_{\alpha}(t)$ are linear and bounded operators, i.e., for any $x \in X$,

$$
\left\|S_{\alpha}(t) x\right\| \leq M\|x\|, \quad\left\|T_{\alpha}(t) x\right\| \leq \frac{\alpha M}{\Gamma(1+\alpha)}\|x\| .
$$

(2) The operators $\left\{S_{\alpha}(t)\right\}_{t \geq 0}$ and $\left\{T_{\alpha}(t)\right\}_{t \geq 0}$ are strongly continuous and compact.

(3) For any $x \in X, \beta \in(0,1)$ and $\theta \in(0,1]$, we have

$$
\begin{aligned}
& A T_{\alpha}(t) x=A^{1-\beta} T_{\alpha}(t) A^{\beta} x, \\
& \left\|A^{\theta} T_{\alpha}(t)\right\| \leq \frac{\alpha c_{\theta} \Gamma(2-\theta)}{t^{\alpha \theta} \Gamma(1+\alpha(1-\theta))}, \quad t \in[0, b] .
\end{aligned}
$$

Definition 3.2 System (1.1) is said to be controllable on the interval $J$ if for every continuous initial function $\phi \in \mathfrak{B}_{h}, x_{1} \in X$, there exists a control $u \in L^{2}(J, U)$ such that the mild solution $x(t)$ of (1.1) satisfies $x(b)=x_{1}$.

To investigate the controllability of system (1.1), we use the following hypotheses:

$\left(H_{1}\right) \quad A$ is the infinitesimal generator of an analytic semigroup of bounded linear operators $T(t), 0 \in \rho(A)$, for $t \geq 0$, there exist constants $M$ such that $|T(t)| \leq M$.

$\left(H_{2}\right)$ The linear operator $W: L^{2}(J, U) \rightarrow X$ defined by

$$
W u=\int_{0}^{b}(b-s)^{\alpha-1} T(b-s) B u(s) d s
$$

has an induced inverse operator $W^{-1}$, which takes values in $L^{2}(J, U) /$ ker $W$ and there exist positive constants $M_{2}, M_{3}$ such that $|B| \leq M_{2}$ and $\left|W^{-1}\right| \leq M_{3}$. 
$\left(H_{3}\right)$ There exist constants $0 \leq \beta<1, c_{0}, c_{1}, c_{2}, L_{g}$ such that $g$ is $X_{\beta}$-valued, $(-A)^{\beta} g$ is continuous, and

(i) $\left\|(-A)^{\beta} g(t, x)\right\| \leq c_{1}\|x\|_{\mathfrak{B}_{h}}+c_{2},(t, x) \in J \times \mathfrak{B}_{h}$;

(ii) $\left\|(-A)^{\beta} g\left(t, x_{1}\right)-(-A)^{\beta} g\left(t, x_{2}\right)\right\| \leq L_{g}\left\|x_{1}-x_{2}\right\|_{\mathfrak{B}_{h}},\left(t, x_{i}\right) \in J \times \mathfrak{B}_{h}, i=1,2$, with

$$
C_{0}=L_{g} l\left[\left\|(-A)^{-\beta}\right\|+\frac{c_{1-\beta} \Gamma(1+\beta) b^{\alpha \beta}}{\beta \Gamma(1+\alpha \beta)}\right]<1 .
$$

$\left(H_{4}\right)$ There exists a constant $d_{k}$ such that $\left\|I_{k}(x)\right\| \leq d_{k}, k=1,2, \ldots, m$ for each $x \in X$.

$\left(H_{5}\right)$ There exist an integrable function $p: J \rightarrow[0,+\infty)$ and a nondecreasing function $\psi$ : $R_{+} \rightarrow(0,+\infty)$ such that $\|F(t, x)\|=\sup \{|f|: f(t) \in F(t, x)\} \leq p(t) \psi\left(\|x\|_{\mathfrak{B}_{h}}\right)$ for almost all $t \in J$ and all $x \in \mathfrak{B}_{h}$.

$\left(H_{6}\right)$ There exists a positive constant $r$ such that

$$
\frac{r}{F_{1}+F_{2} r+F_{3} \psi\left(l r+\|\phi\|_{\mathfrak{B}_{h}}+l M|\phi(0)|\right)}>1
$$

where

$$
\begin{aligned}
F_{1}= & K_{1}+\frac{M M_{2} M_{3} b^{\alpha}}{\Gamma(1+\alpha)} \cdot\left\{\left|x_{1}\right|+M|\phi(0)|+K_{1}\right\}, \\
F_{2}= & K_{2}+\frac{M M_{2} M_{3} b^{\alpha}}{\Gamma(1+\alpha)} \cdot K_{2}, \quad F_{3}=\left[1+\frac{M M_{2} M_{3} b^{\alpha}}{\Gamma(1+\alpha)}\right] \frac{b^{\alpha} M}{\Gamma(1+\alpha)} \sup _{s \in J} p(s), \\
K_{1}= & M\left[\left\|(-A)^{-\beta}\right\|\left(c_{1}\|\phi\|_{\mathfrak{B}_{h}}+c_{2}\right)\right] \\
& +\left[\left\|(-A)^{-\beta}\right\|+\frac{c_{1-\beta} \Gamma(1+\beta)}{\Gamma(1+\alpha \beta)} \cdot \frac{b^{\alpha \beta}}{\beta}\right]\left(c_{1}\|\phi\|_{\mathfrak{B}_{h}}+c_{1} l M|\phi(0)|+c_{2}\right) \\
& +M \sum_{k=1}^{m} d_{k}, \\
K_{2}= & \left\|(-A)^{-\beta}\right\| c_{1} l+\frac{c_{1-\beta} \Gamma(1+\beta)}{\Gamma(1+\alpha \beta)} \cdot \frac{b^{\alpha \beta}}{\beta} c_{1} l .
\end{aligned}
$$

Lemma 3.2 (Lasota and Opial [35]) Let I be a compact real interval and X be a Banach space. Let $F$ be a multivalued map satisfying $\left(H_{5}\right)$ and let $\Gamma$ be a linear continuous mapping from $L^{1}(I, X)$ to $C(I, X)$. Then the operator

$$
\Gamma \circ S_{F}: C(I, X) \rightarrow B C C(C(I, X)), \quad x \rightarrow\left(\Gamma \circ S_{F}\right)(x)=\Gamma\left(S_{F, x}\right),
$$

is a closed graph operator in $C(I, X) \times C(I, X)$.

Lemma 3.3 $[17,27]$ Suppose $x \in \mathfrak{B}_{b}$, then for $t \in J, x_{t} \in \mathfrak{B}_{h}$. Moreover,

$$
l|x(t)| \leq\left\|x_{t}\right\|_{\mathfrak{B}_{h}} \leq l \sup _{s \in[0, t]}|x(s)|+\left\|x_{0}\right\|_{\mathfrak{B}_{h}},
$$

where $l=\int_{-\infty}^{0} h(s) d s<+\infty$. 
Now, consider the multivalued map $\mathfrak{L}: \mathfrak{B}_{b} \rightarrow 2^{\mathfrak{B}_{b}}$ defined by $\mathfrak{L} x$ the set of $\rho \in \mathfrak{B}_{b}$ such that

$$
\rho(t)=\left\{\begin{array}{l}
\phi(t), \quad t \in(-\infty, 0], \\
S_{\alpha}(t)[\phi(0)-g(0, \phi)]+g\left(t, x_{t}\right)+\int_{0}^{t}(t-s)^{\alpha-1} A T_{\alpha}(t-s) g\left(s, x_{s}\right) d s \\
\quad+\int_{0}^{t}(t-s)^{\alpha-1} T_{\alpha}(t-s) f(s) d s+\sum_{0<t_{k}<t} S_{\alpha}\left(t-t_{k}\right) I_{k}\left(x\left(t_{k}^{-}\right)\right) \\
\quad+\int_{0}^{t}(t-\eta)^{\alpha-1} T_{\alpha}(t-\eta) B W^{-1}\left[x_{1}-S_{\alpha}(b)[\phi(0)-g(0, \phi)]\right. \\
\quad-g\left(b, y_{b}+\bar{\phi}_{b}\right)-\int_{0}^{b}(b-s)^{\alpha-1} A T_{\alpha}(b-s) g\left(s, x_{s}\right) d s \\
\quad-\int_{0}^{b}(b-s)^{\alpha-1} T_{\alpha}(b-s) f(s) d s \\
\left.\quad-\sum_{k=1}^{m} S_{\alpha}\left(b-t_{k}\right) I_{k}\left(x\left(t_{k}^{-}\right)\right)\right](\eta) d \eta, \quad t \in J,
\end{array}\right.
$$

where $f \in S_{F, x}$.

We shall show that the operator $\mathfrak{L}$ has fixed points, which are then a solution of system (1.1). For $\phi \in \mathfrak{B}_{h}$, we define $\bar{\phi}$ by

$$
\bar{\phi}(t)= \begin{cases}\phi(t), & -\infty<t \leq 0, \\ S_{\alpha}(t) \phi(0), & 0 \leq t \leq b,\end{cases}
$$

then $\bar{\phi} \in \mathfrak{B}_{b}$. Set

$$
x(t)=y(t)+\bar{\phi}(t), \quad-\infty<t \leq b .
$$

It is clear that $x$ satisfies (3.1) if and only if $y$ satisfies $y_{0}=0$ and

$$
\begin{aligned}
y(t)= & -S_{\alpha}(t) g(0, \phi)+g\left(t, y_{t}+\bar{\phi}_{t}\right)+\int_{0}^{t}(t-s)^{\alpha-1} A T_{\alpha}(t-s) g\left(s, y_{s}+\bar{\phi}_{s}\right) d s \\
& +\int_{0}^{t}(t-s)^{\alpha-1} T_{\alpha}(t-s) f(s) d s+\sum_{0<t_{k}<t} S_{\alpha}\left(t-t_{k}\right) I_{k}\left(y\left(t_{k}^{-}\right)+\bar{\phi}\left(t_{k}^{-}\right)\right) \\
& +\int_{0}^{t}(t-\eta)^{\alpha-1} T_{\alpha}(t-\eta) B W^{-1}\left[x_{1}-S_{\alpha}(b)[\phi(0)-g(0, \phi)]-g\left(b, y_{b}+\bar{\phi}_{b}\right)\right. \\
& -\int_{0}^{b}(b-s)^{\alpha-1} A T_{\alpha}(b-s) g\left(s, y_{s}+\bar{\phi}_{s}\right) d s \\
& -\int_{0}^{b}(b-s)^{\alpha-1} T_{\alpha}(b-s) f(s) d s \\
& \left.-\sum_{k=1}^{m} S_{\alpha}\left(b-t_{k}\right) I_{k}\left(y\left(t_{k}^{-}\right)+\bar{\phi}\left(t_{k}^{-}\right)\right)\right](\eta) d \eta, \quad t \in J .
\end{aligned}
$$

Let $\mathfrak{B}_{b}^{0}=\left\{y \in \mathfrak{B}_{b}: y_{0}=0 \in \mathfrak{B}_{h}\right\}$. For any $y \in \mathfrak{B}_{b}^{0}$,

$$
\|y\|_{b}=\left\|y_{0}\right\|_{\mathfrak{B}_{h}}+\sup \{|y(s)|: 0 \leq s \leq b\}=\sup \{|y(s)|: 0 \leq s \leq b\} .
$$


Thus $\left(\mathfrak{B}_{b}^{0},\|\cdot\|_{b}\right)$ is a Banach space. Set $\mathfrak{B}_{q}=\left\{y \in \mathfrak{B}_{b}^{0}:\|y\|_{b} \leq q\right\}$ for some $q \geq 0$, then $\mathfrak{B}_{q} \subseteq \mathfrak{B}_{b}^{0}$ is uniformly bounded, for any $y \in \mathfrak{B}_{q}$, and from Lemma 3.3, we have

$$
\begin{aligned}
\left\|y_{t}+\bar{\phi}_{t}\right\|_{\mathfrak{B}_{h}} & \leq\left\|y_{t}\right\|_{\mathfrak{B}_{h}}+\left\|\bar{\phi}_{t}\right\|_{\mathfrak{B}_{h}} \\
& \leq l \sup _{s \in[0, t]}|y(s)|+\left\|y_{0}\right\|_{\mathfrak{B}_{h}}+l \sup _{s \in[0, t]}|\bar{\phi}(s)|+\left\|\bar{\phi}_{0}\right\|_{\mathfrak{B}_{h}} \\
& \leq l(q+M|\phi(0)|)+\|\phi\|_{\mathfrak{B}_{h}}=q^{\prime} .
\end{aligned}
$$

Define the multivalued map $\Phi: \mathfrak{B}_{b}^{0} \rightarrow 2^{\mathfrak{B}_{b}^{0}}$ defined by $\Phi y$, the set of $\bar{\rho} \in \mathfrak{B}_{b}^{0}$ such that

$$
\bar{\rho}(t)=\left\{\begin{aligned}
0, & t \in(-\infty, 0], \\
- & S_{\alpha}(t) g(0, \phi)+g\left(t, y_{t}+\bar{\phi}_{t}\right)+\int_{0}^{t}(t-s)^{\alpha-1} A T_{\alpha}(t-s) g\left(s, y_{s}+\bar{\phi}_{s}\right) d s \\
& +\int_{0}^{t}(t-s)^{\alpha-1} T_{\alpha}(t-s) f(s) d s+\sum_{0<t_{k}<t} S_{\alpha}\left(t-t_{k}\right) I_{k}\left(y\left(t_{k}^{-}\right)+\bar{\phi}\left(t_{k}^{-}\right)\right) \\
& +\int_{0}^{t}(t-\eta)^{\alpha-1} T_{\alpha}(t-\eta) B W^{-1}\left[x_{1}-S_{\alpha}(b)[\phi(0)-g(0, \phi)]\right. \\
& -g\left(b, y_{b}+\bar{\phi}_{b}\right)-\int_{0}^{b}(b-s)^{\alpha-1} A T_{\alpha}(b-s) g\left(s, y_{s}+\bar{\phi}_{s}\right) d s \\
& -\int_{0}^{b}(b-s)^{\alpha-1} T_{\alpha}(b-s) f(s) d s \\
& \left.-\sum_{k=1}^{m} S_{\alpha}\left(b-t_{k}\right) I_{k}\left(y\left(t_{k}^{-}\right)+\bar{\phi}\left(t_{k}^{-}\right)\right)\right](\eta) d \eta, \quad t \in J .
\end{aligned}\right.
$$

Now we decompose $\Phi$ as $\Phi_{1}+\Phi_{2}$, where

$$
\begin{aligned}
\Phi_{1} y(t)= & -S_{\alpha}(t) g(0, \phi)+g\left(t, y_{t}+\bar{\phi}_{t}\right)+\int_{0}^{t}(t-s)^{\alpha-1} A T_{\alpha}(t-s) g\left(s, y_{s}+\bar{\phi}_{s}\right) d s, \\
\Phi_{2} y(t)= & \int_{0}^{t}(t-s)^{\alpha-1} T_{\alpha}(t-s) f(s) d s+\sum_{0<t_{k}<t} S_{\alpha}\left(t-t_{k}\right) I_{k}\left(y\left(t_{k}^{-}\right)+\bar{\phi}\left(t_{k}^{-}\right)\right) \\
& +\int_{0}^{t}(t-\eta)^{\alpha-1} T_{\alpha}(t-\eta) B W^{-1}\left[x_{1}-S_{\alpha}(b)[\phi(0)-g(0, \phi)]-g\left(b, y_{b}+\bar{\phi}_{b}\right)\right. \\
& -\int_{0}^{b}(b-s)^{\alpha-1} A T_{\alpha}(b-s) g\left(s, y_{s}+\bar{\phi}_{s}\right) d s \\
& -\int_{0}^{b}(b-s)^{\alpha-1} T_{\alpha}(b-s) f(s) d s \\
& \left.-\sum_{k=1}^{m} S_{\alpha}\left(b-t_{k}\right) I_{k}\left(y\left(t_{k}^{-}\right)+\bar{\phi}\left(t_{k}^{-}\right)\right)\right](\eta) d \eta, \quad t \in J .
\end{aligned}
$$

Theorem 3.4 Assume that hypotheses $\left(H_{1}\right)-\left(H_{6}\right)$ hold, then system (1.1) is controllable on $J$.

Proof We divide the proof into several steps.

Step 1 . We remark that $\Phi_{1}$ for each $y \in \mathfrak{B}_{b}^{0}$ has closed, convex values on $\mathfrak{B}_{b}^{0}$. Next we show that $\Phi_{1}$ has bounded values for bounded in $\mathfrak{B}_{b}^{0}$. To show this, let $\mathfrak{B}_{q}=\left\{y \in \mathfrak{B}_{b}^{0}\right.$ : $\left.\|y\|_{b} \leq q\right\}$ for some $q>0$. Then, for any $y \in \mathfrak{B}_{q}$, one has

$$
\begin{aligned}
\left\|\Phi_{1} y(t)\right\| \leq & M\|g(0, \phi)\|+\left\|(-A)^{-\beta}\right\|\left[c_{1}\left\|y_{t}+\bar{\phi}_{t}\right\|_{\mathfrak{B}_{h}}+c_{2}\right] \\
& +\int_{0}^{t}\left\|(t-s)^{\alpha-1} A^{1-\beta} T_{\alpha}(t-s) A^{\beta} g\left(s, y_{s}+\bar{\phi}_{s}\right)\right\| d s
\end{aligned}
$$




$$
\begin{gathered}
\leq M\|g(0, \phi)\|+\left\|(-A)^{-\beta}\right\|\left(c_{1} q^{\prime}+c_{2}\right) \\
+\frac{\left(c_{1} q^{\prime}+c_{2}\right) \alpha c_{1-\beta} \Gamma(1+\beta) b^{\alpha \beta}}{\Gamma(1+\alpha \beta) \alpha \beta} .
\end{gathered}
$$

Hence $\Phi_{1}$ is bounded.

Step 2. $\Phi_{2} y$ is convex for each $y \in \mathfrak{B}_{b}^{0}$.

In fact, if $\bar{\rho}_{1}, \bar{\rho}_{2}$ belong to $\Phi_{2} y$, then there exist $f_{1}, f_{2} \in S_{F, y}$ such that, for each $t \in J$, we have

$$
\begin{aligned}
\bar{\rho}_{i}(t)= & \int_{0}^{t}(t-s)^{\alpha-1} T_{\alpha}(t-s) f_{i}(s) d s+\sum_{0<t_{k}<t} S_{\alpha}\left(t-t_{k}\right) I_{k}\left(y\left(t_{k}^{-}\right)+\bar{\phi}\left(t_{k}^{-}\right)\right) \\
& +\int_{0}^{t}(t-\eta)^{\alpha-1} T_{\alpha}(t-\eta) B W^{-1}\left[x_{1}-S_{\alpha}(b)[\phi(0)-g(0, \phi)]-g\left(b, y_{b}+\bar{\phi}_{b}\right)\right. \\
& -\int_{0}^{b}(b-s)^{\alpha-1} A T_{\alpha}(b-s) g\left(s, y_{s}+\bar{\phi}_{s}\right) d s-\int_{0}^{b}(b-s)^{\alpha-1} T_{\alpha}(b-s) f_{i}(s) d s \\
& \left.-\sum_{k=1}^{m} S_{\alpha}\left(b-t_{k}\right) I_{k}\left(y\left(t_{k}^{-}\right)+\bar{\phi}\left(t_{k}^{-}\right)\right)\right](\eta) d \eta, \quad i=1,2 .
\end{aligned}
$$

Let $\mu \in[0,1]$, since the operators $B$ and $W^{-1}$ are linear, we have

$$
\begin{aligned}
& \left(\mu \bar{\rho}_{1}+(1-\mu) \bar{\rho}_{2}\right)(t) \\
& =\int_{0}^{t}(t-s)^{\alpha-1} T_{\alpha}(t-s)\left[\mu f_{1}(s)+(1-\mu) f_{2}(s)\right] d s+\sum_{0<t_{k}<t} S_{\alpha}\left(t-t_{k}\right) I_{k}\left(y\left(t_{k}^{-}\right)+\bar{\phi}\left(t_{k}^{-}\right)\right) \\
& \quad+\int_{0}^{t}(t-\eta)^{\alpha-1} T_{\alpha}(t-\eta) B W^{-1}\left[x_{1}-S_{\alpha}(b)[\phi(0)-g(0, \phi)]-g\left(b, y_{b}+\bar{\phi}_{b}\right)\right. \\
& \quad-\int_{0}^{b}(b-s)^{\alpha-1} A T_{\alpha}(b-s) g\left(s, y_{s}+\bar{\phi}_{s}\right) d s \\
& \quad-\int_{0}^{b}(b-s)^{\alpha-1} T_{\alpha}(b-s)\left[\mu f_{1}(s)+(1-\mu) f_{2}(s)\right] d s \\
& \left.\quad-\sum_{k=1}^{m} S_{\alpha}\left(b-t_{k}\right) I_{k}\left(y\left(t_{k}^{-}\right)+\bar{\phi}\left(t_{k}^{-}\right)\right)\right](\eta) d \eta .
\end{aligned}
$$

Since $S_{F, y}$ is convex (because $F$ has convex values), we have $\left(\mu \bar{\rho}_{1}+(1-\mu) \bar{\rho}_{2}\right) \in \Phi_{2} y$.

Step 3 . We will prove that the operator $\Phi_{1}$ is a contraction operator on $\mathfrak{B}_{b}^{0}$. Let $u, v \in \mathfrak{B}_{b}^{0}$; we have

$$
\begin{aligned}
& \left\|\Phi_{1} u(t)-\Phi_{1} v(t)\right\| \\
& \quad \leq\left\|g\left(t, u_{t}+\bar{\phi}_{t}\right)-g\left(t, v_{t}+\bar{\phi}_{t}\right)\right\| \\
& \quad+\int_{0}^{t}\left\|(t-s)^{\alpha-1} A T_{\alpha}(t-s)\left[g\left(s, u_{s}+\bar{\phi}_{s}\right)-g\left(s, v_{s}+\bar{\phi}_{s}\right)\right]\right\| d s \\
& \quad \leq\left\|(-A)^{-\beta}\right\| L_{g}\left\|u_{t}-v_{t}\right\|_{\mathfrak{B}_{h}}+L_{g}\left\|u_{t}-v_{t}\right\|_{\mathfrak{B}_{h}} \int_{0}^{t}(t-s)^{\alpha-1}\left\|A^{1-\beta} T_{\alpha}(t-s)\right\| d s
\end{aligned}
$$




$$
\begin{aligned}
\leq & \left\|(-A)^{-\beta}\right\| L_{g}\left\|u_{t}-v_{t}\right\|_{\mathfrak{B}_{h}}+L_{g}\left\|u_{t}-v_{t}\right\|_{\mathfrak{B}_{h}} \int_{0}^{t}(t-s)^{\alpha-1} \frac{\alpha c_{1-\beta} \Gamma(1+\beta)}{(t-s)^{\alpha(1-\beta)} \Gamma(1+\alpha \beta)} d s \\
\leq & \left\|(-A)^{-\beta}\right\| L_{g}\left\|u_{t}-v_{t}\right\|_{\mathfrak{B}_{h}}+L_{g}\left\|u_{t}-v_{t}\right\|_{\mathfrak{B}_{h}} \frac{c_{1-\beta} \Gamma(1+\beta) b^{\alpha \beta}}{\beta \Gamma(1+\alpha \beta)} \\
\leq & \left\|(-A)^{-\beta}\right\| L_{g}\left[l \sup _{s \in[0, t]}|u(s)-v(s)|+\left\|u_{0}-v_{0}\right\|_{\mathfrak{B}_{h}}\right] \\
& +L_{g}\left[l \sup _{s \in[0, t]}|u(s)-v(s)|+\left\|u_{0}-v_{0}\right\|_{\mathfrak{B}_{h}}\right] \frac{c_{1-\beta} \Gamma(1+\beta) b^{\alpha \beta}}{\beta \Gamma(1+\alpha \beta)} \\
\leq & L_{g} l\left[\left\|(-A)^{-\beta}\right\|+\frac{c_{1-\beta} \Gamma(1+\beta) b^{\alpha \beta}}{\beta \Gamma(1+\alpha \beta)}\right] \sup _{s \in[0, b]}|u(s)-v(s)|,
\end{aligned}
$$

since $\left\|u_{0}-v_{0}\right\|_{\mathfrak{B}_{h}}=0$, taking the supremum over $t,\left\|\Phi_{1} u-\Phi_{1} v\right\| \leq C_{0}\|u-v\|$, where

$$
C_{0}=L_{g} l\left[\left\|(-A)^{-\beta}\right\|+\frac{c_{1-\beta} \Gamma(1+\beta) b^{\alpha \beta}}{\beta \Gamma(1+\alpha \beta)}\right]<1 .
$$

Thus $\Phi_{1}$ is a contraction on $\mathfrak{B}_{b}^{0}$.

Step 4 . Next we show that the operator $\Phi_{2}$ is completely continuous. First, we prove that $\Phi_{2}$ maps a bounded set into a bounded set in $\mathfrak{B}_{b}^{0}$. Indeed, it is enough to show that there exists a positive constant $\Lambda$ such that, for each $\bar{\rho} \in \Phi_{2} y, y \in \mathfrak{B}_{q}=\left\{y \in \mathfrak{B}_{b}^{0}:\|y\|_{b} \leq q\right\}$, one has $\|\bar{\rho}\|_{b} \leq \Lambda$. If $\bar{\rho} \in \Phi_{2} y$, then there exists $f \in S_{F, y}$, such that, for each $t \in J$,

$$
\begin{aligned}
\bar{\rho}(t)= & \int_{0}^{t}(t-s)^{\alpha-1} T_{\alpha}(t-s) f(s) d s+\sum_{0<t_{k}<t} S_{\alpha}\left(t-t_{k}\right) I_{k}\left(y\left(t_{k}^{-}\right)+\bar{\phi}\left(t_{k}^{-}\right)\right) \\
& +\int_{0}^{t}(t-\eta)^{\alpha-1} T_{\alpha}(t-\eta) B W^{-1}\left[x_{1}-S_{\alpha}(b)[\phi(0)-g(0, \phi)]-g\left(b, y_{b}+\bar{\phi}_{b}\right)\right. \\
& -\int_{0}^{b}(b-s)^{\alpha-1} A T_{\alpha}(b-s) g\left(s, y_{s}+\bar{\phi}_{s}\right) d s-\int_{0}^{b}(b-s)^{\alpha-1} T_{\alpha}(b-s) f(s) d s \\
& \left.-\sum_{k=1}^{m} S_{\alpha}\left(b-t_{k}\right) I_{k}\left(y\left(t_{k}^{-}\right)+\bar{\phi}\left(t_{k}^{-}\right)\right)\right](\eta) d \eta, \quad t \in J .
\end{aligned}
$$

We have for $t \in J$

$$
\begin{aligned}
|\bar{\rho}(t)| \leq & \int_{0}^{t}(t-s)^{\alpha-1}\left|T_{\alpha}(t-s) f(s)\right| d s+\sum_{0<t_{k}<t}\left|S_{\alpha}\left(t-t_{k}\right) I_{k}\left(y\left(t_{k}^{-}\right)+\bar{\phi}\left(t_{k}^{-}\right)\right)\right| \\
& +\int_{0}^{t} \mid(t-\eta)^{\alpha-1} T_{\alpha}(t-\eta) B W^{-1}\left[x_{1}-S_{\alpha}(b)[\phi(0)-g(0, \phi)]-g\left(b, y_{b}+\bar{\phi}_{b}\right)\right. \\
& -\int_{0}^{b}(b-s)^{\alpha-1} A T_{\alpha}(b-s) g\left(s, y_{s}+\bar{\phi}_{s}\right) d s-\int_{0}^{b}(b-s)^{\alpha-1} T_{\alpha}(b-s) f(s) d s \\
& \left.-\sum_{k=1}^{m} S_{\alpha}\left(b-t_{k}\right) I_{k}\left(y\left(t_{k}^{-}\right)+\bar{\phi}\left(t_{k}^{-}\right)\right)\right] \mid(\eta) d \eta \\
\leq & \int_{0}^{t}(t-s)^{\alpha-1} \frac{\alpha M}{\Gamma(1+\alpha)} p(s) \psi\left(\left\|y_{s}+\bar{\phi}_{s}\right\|_{\mathfrak{B}_{h}}\right) d s+M \sum_{k=1}^{m} d_{k}
\end{aligned}
$$




$$
\begin{aligned}
& +\frac{\alpha M M_{2} M_{3}}{\Gamma(1+\alpha)} \int_{0}^{t}(t-\eta)^{\alpha-1} \mid x_{1}-S_{\alpha}(b)[\phi(0)-g(0, \phi)]-g\left(b, y_{b}+\bar{\phi}_{b}\right) \\
& -\int_{0}^{b}(b-s)^{\alpha-1} A T_{\alpha}(b-s) g\left(s, y_{s}+\bar{\phi}_{s}\right) d s-\int_{0}^{b}(b-s)^{\alpha-1} T_{\alpha}(b-s) f(s) d s \\
& -\sum_{k=1}^{m} S_{\alpha}\left(b-t_{k}\right) I_{k}\left(y\left(t_{k}^{-}\right)+\bar{\phi}\left(t_{k}^{-}\right)\right) \mid(\eta) d \eta \\
& \leq \sup _{s \in J} p(s) \frac{b^{\alpha} M}{\Gamma(1+\alpha)} \sup _{y \in\left[0, q^{\prime}\right]} \psi(y)+M \sum_{k=1}^{m} d_{k} \\
& +\frac{\alpha M M_{2} M_{3}}{\Gamma(1+\alpha)} \int_{0}^{t}(t-\eta)^{\alpha-1}\left[\left|x_{1}\right|+M\left(\phi(0)+\left\|(-A)^{-\beta}\right\|\left(c_{1}\|\phi\|_{\mathfrak{B}_{h}}+c_{2}\right)\right)\right. \\
& +\left\|(-A)^{-\beta}\right\|\left(c_{1}\left\|y_{b}+\bar{\phi}_{b}\right\|_{\mathfrak{B}_{h}}+c_{2}\right) \\
& +\int_{0}^{b} \frac{\alpha c_{1-\beta} \Gamma(1+\beta)}{\Gamma(1+\alpha \beta)}(b-s)^{\alpha \beta-1}\left(c_{1}\left\|y_{s}+\bar{\phi}_{s}\right\|_{\mathfrak{B}_{h}}+c_{2}\right) d s \\
& \left.+\int_{0}^{b}(b-s)^{\alpha-1} \frac{\alpha M}{\Gamma(1+\alpha)} p(s) \psi\left(\left\|y_{s}+\bar{\phi}_{s}\right\|_{\mathfrak{B}_{h}}\right) d s+M \sum_{k=1}^{m} d_{k}\right](\eta) d \eta \\
& \leq \sup _{s \in J} p(s) \frac{b^{\alpha} M}{\Gamma(1+\alpha)} \sup _{y \in\left[0, q^{\prime}\right]} \psi(y)+M \sum_{k=1}^{m} d_{k} \\
& +\frac{\alpha M M_{2} M_{3}}{\Gamma(1+\alpha)}\left[\left|x_{1}\right|+M\left(\phi(0)+\left\|(-A)^{-\beta}\right\|\left(c_{1}\|\phi\|_{\mathfrak{B}_{h}}+c_{2}\right)\right)\right. \\
& +\left\|(-A)^{-\beta}\right\|\left(c_{1} q^{\prime}+c_{2}\right)+\left(c_{1} q^{\prime}+c_{2}\right) \frac{c_{1-\beta} \Gamma(1+\beta) b^{\alpha \beta}}{\beta \Gamma(1+\alpha \beta)} \\
& \left.+\sup _{s \in J} p(s) \frac{b^{\alpha} M}{\Gamma(1+\alpha)} \sup _{y \in\left[0, q^{\prime}\right]} \psi(y)+M \sum_{k=1}^{m} d_{k}\right] \cdot \frac{b^{\alpha}}{\alpha} \\
& =\Lambda \text {, }
\end{aligned}
$$

then, for each $\bar{\rho} \in \Phi_{2}\left(\mathfrak{B}_{q}\right)$, we have

$$
\|\bar{\rho}\|_{b} \leq \Lambda
$$

Step 5. Next, we show that $\Phi_{2}$ maps bounded sets into equicontinuous sets of $\mathfrak{B}_{b}^{0}$.

Let $r_{1}, r_{2} \in J, 0<r_{1}<r_{2} \leq b$, for each $y \in \mathfrak{B}_{q}=\left\{y \in \mathfrak{B}_{b}^{0}:\|y\|_{b} \leq q\right\}$ and $\bar{\rho} \in \Phi_{2} y$, then there exists $f \in S_{F, y}$, such that, for each $t \in J$,

$$
\begin{aligned}
\bar{\rho}(t)= & \int_{0}^{t}(t-s)^{\alpha-1} T_{\alpha}(t-s) f(s) d s+\sum_{0<t_{k}<t} S_{\alpha}\left(t-t_{k}\right) I_{k}\left(y\left(t_{k}^{-}\right)+\bar{\phi}\left(t_{k}^{-}\right)\right) \\
& +\int_{0}^{t}(t-\eta)^{\alpha-1} T_{\alpha}(t-\eta) B W^{-1}\left[x_{1}-S_{\alpha}(b)[\phi(0)-g(0, \phi)]-g\left(b, y_{b}+\bar{\phi}_{b}\right)\right. \\
& -\int_{0}^{b}(b-s)^{\alpha-1} A T_{\alpha}(b-s) g\left(s, y_{s}+\bar{\phi}_{s}\right) d s
\end{aligned}
$$




$$
\begin{aligned}
& -\int_{0}^{b}(b-s)^{\alpha-1} T_{\alpha}(b-s) f(s) d s \\
& \left.-\sum_{k=1}^{m} S_{\alpha}\left(b-t_{k}\right) I_{k}\left(y\left(t_{k}^{-}\right)+\bar{\phi}\left(t_{k}^{-}\right)\right)\right](\eta) d \eta, \quad t \in J
\end{aligned}
$$

Let $r_{1}, r_{2} \in J-\left\{t_{1}, t_{2}, \ldots, t_{m}\right\}$, we have

$$
\begin{aligned}
& \left\|\bar{\rho}\left(r_{1}\right)-\bar{\rho}\left(r_{2}\right)\right\| \\
& \leq\left|\int_{0}^{r_{1}}\left(r_{1}-s\right)^{\alpha-1}\left[T_{\alpha}\left(r_{2}-s\right)-T_{\alpha}\left(r_{1}-s\right)\right] f(s) d s\right| \\
& +\left|\int_{0}^{r_{1}}\left[\left(r_{2}-s\right)^{\alpha-1}-\left(r_{1}-s\right)^{\alpha-1}\right] T_{\alpha}\left(r_{2}-s\right) f(s) d s\right| \\
& +\left|\int_{r_{1}}^{r_{2}}\left(r_{2}-s\right)^{\alpha-1} T_{\alpha}\left(r_{2}-s\right) f(s) d s\right| \\
& +\left|\sum_{0<t_{k}<r_{1}}\left[S_{\alpha}\left(r_{2}-t_{k}\right)-S_{\alpha}\left(r_{1}-t_{k}\right)\right] I_{k}\left(y\left(t_{k}^{-}\right)+\bar{\phi}\left(t_{k}^{-}\right)\right)\right| \\
& +\left|\sum_{r_{1} \leq t_{k}<r_{2}} S_{\alpha}\left(r_{2}-t_{k}\right) I_{k}\left(y\left(t_{k}^{-}\right)+\bar{\phi}\left(t_{k}^{-}\right)\right)\right| \\
& +\left|\int_{0}^{r_{1}}\left(r_{1}-\eta\right)^{\alpha-1}\left[T_{\alpha}\left(r_{2}-\eta\right)-T_{\alpha}\left(r_{1}-\eta\right)\right] B u(\eta) d \eta\right| \\
& +\left|\int_{0}^{r_{1}}\left[\left(r_{2}-\eta\right)^{\alpha-1}-\left(r_{1}-\eta\right)^{\alpha-1}\right] T_{\alpha}\left(r_{2}-\eta\right) B u(\eta) d \eta\right| \\
& +\left|\int_{r_{1}}^{r_{2}}\left(r_{2}-\eta\right)^{\alpha-1} T_{\alpha}\left(r_{2}-\eta\right) B u(\eta) d \eta\right| \\
& \leq \int_{0}^{r_{1}}\left(r_{1}-s\right)^{\alpha-1}\left\|T_{\alpha}\left(r_{2}-s\right)-T_{\alpha}\left(r_{1}-s\right)\right\|\|f(s)\| d s \\
& +\int_{0}^{r_{1}}\left|\left(r_{2}-s\right)^{\alpha-1}-\left(r_{1}-s\right)^{\alpha-1}\right|\left|T_{\alpha}\left(r_{2}-s\right)\right| \mid f(s) \| d s \\
& +\int_{r_{1}}^{r_{2}}\left(r_{2}-s\right)^{\alpha-1}\left\|T_{\alpha}\left(r_{2}-s\right)\right\|\|f(s)\| d s \\
& +\sum_{0<t_{k}<r_{1}}\left|S_{\alpha}\left(r_{2}-t_{k}\right)-S_{\alpha}\left(r_{1}-t_{k}\right)\right| d_{k}+\sum_{r_{1} \leq t_{k}<r_{2}} M d_{k} \\
& +\int_{0}^{r_{1}}\left(r_{1}-\eta\right)^{\alpha-1}\left\|T_{\alpha}\left(r_{2}-\eta\right)-T_{\alpha}\left(r_{1}-\eta\right)\right\|\|B u(\eta)\| d \eta \\
& +\int_{0}^{r_{1}}\left[\left(r_{2}-\eta\right)^{\alpha-1}-\left(r_{1}-\eta\right)^{\alpha-1}\right]\left\|T_{\alpha}\left(r_{2}-\eta\right)\right\|\|B u(\eta)\| d \eta \\
& +\int_{r_{1}}^{r_{2}}\left(r_{2}-\eta\right)^{\alpha-1}\left\|T_{\alpha}\left(r_{2}-\eta\right)\right\|\|B u(\eta)\| d \eta .
\end{aligned}
$$

As $r_{2} \rightarrow r_{1}$, the right-hand side of the above inequality tends to zero, thus the set $\left\{\Phi_{2} y\right.$ : $\left.y \in \mathfrak{B}_{q}\right\}$ is equicontinuous. This proves the equicontinuity in the case where $t \neq t_{i}, i=$ $1,2, \ldots, m$. Similarly one can prove that $t=t_{i}$. The equicontinuities for the other cases, 
$r_{1}<r_{2} \leq 0$ or $r_{1} \leq 0 \leq r_{2} \leq b$, are very simple. As a consequence of the Arzela-Ascoli theorem, $\Phi_{2}$ is completely continuous.

Step 6. $\Phi_{2}$ has a closed graph.

Let $y^{(n)} \rightarrow y^{*}, \bar{\rho}_{n} \in \Phi_{2}\left(y^{(n)}\right)$ and $\bar{\rho}_{n} \rightarrow \bar{\rho}_{*}$. We shall prove that $\bar{\rho}_{*} \in \Phi_{2}\left(y^{*}\right)$. Indeed, $\bar{\rho}_{n} \in$ $\Phi_{2}\left(y^{(n)}\right)$ means that there exists $f_{n} \in S_{F, y^{(n)}}$, such that

$$
\begin{aligned}
\bar{\rho}_{n}(t)= & \int_{0}^{t}(t-s)^{\alpha-1} T_{\alpha}(t-s) f_{n}(s) d s+\sum_{0<t_{k}<t} S_{\alpha}\left(t-t_{k}\right) I_{k}\left(y^{(n)}\left(t_{k}^{-}\right)+\bar{\phi}\left(t_{k}^{-}\right)\right) \\
& +\int_{0}^{t}(t-\eta)^{\alpha-1} T_{\alpha}(t-\eta) B W^{-1}\left[x_{1}-S_{\alpha}(b)[\phi(0)-g(0, \phi)]-g\left(b, y_{b}^{(n)}+\bar{\phi}_{b}\right)\right. \\
& -\int_{0}^{b}(b-s)^{\alpha-1} A T_{\alpha}(b-s) g\left(s, y_{s}^{(n)}+\bar{\phi}_{s}\right) d s-\int_{0}^{b}(b-s)^{\alpha-1} T_{\alpha}(b-s) f_{n}(s) d s \\
& \left.-\sum_{k=1}^{m} S_{\alpha}\left(b-t_{k}\right) I_{k}\left(y^{(n)}\left(t_{k}^{-}\right)+\bar{\phi}\left(t_{k}^{-}\right)\right)\right](\eta) d \eta, \quad t \in J .
\end{aligned}
$$

We must prove that there exists $f_{*} \in S_{F, y^{*}}$ such that

$$
\begin{aligned}
\bar{\rho}_{*}(t)= & \int_{0}^{t}(t-s)^{\alpha-1} T_{\alpha}(t-s) f_{*}(s) d s+\sum_{0<t_{k}<t} S_{\alpha}\left(t-t_{k}\right) I_{k}\left(y^{(*)}\left(t_{k}^{-}\right)+\bar{\phi}\left(t_{k}^{-}\right)\right) \\
& +\int_{0}^{t}(t-\eta)^{\alpha-1} T_{\alpha}(t-\eta) B W^{-1}\left[x_{1}-S_{\alpha}(b)[\phi(0)-g(0, \phi)]-g\left(b, y_{b}^{(*)}+\bar{\phi}_{b}\right)\right. \\
& -\int_{0}^{b}(b-s)^{\alpha-1} A T_{\alpha}(b-s) g\left(s, y_{s}^{(*)}+\bar{\phi}_{s}\right) d s-\int_{0}^{b}(b-s)^{\alpha-1} T_{\alpha}(b-s) f_{*}(s) d s \\
& \left.-\sum_{k=1}^{m} S_{\alpha}\left(b-t_{k}\right) I_{k}\left(y^{(*)}\left(t_{k}^{-}\right)+\bar{\phi}\left(t_{k}^{-}\right)\right)\right](\eta) d \eta, \quad t \in J ;
\end{aligned}
$$

since $I_{k}, k=0,1,2, \ldots, m$ are continuous, we obtain

$$
\begin{aligned}
\|\left\{\bar{\rho}_{n}(t)\right. & -\sum_{0<t_{k}<t} S_{\alpha}\left(t-t_{k}\right) I_{k}\left(y^{(n)}\left(t_{k}^{-}\right)+\bar{\phi}\left(t_{k}^{-}\right)\right) \\
& -\int_{0}^{t}(t-\eta)^{\alpha-1} T_{\alpha}(t-\eta) B W^{-1}\left[x_{1}-S_{\alpha}(b)[\phi(0)-g(0, \phi)]-g\left(b, y_{b}^{(n)}+\bar{\phi}_{b}\right)\right. \\
& -\int_{0}^{b}(b-s)^{\alpha-1} A T_{\alpha}(b-s) g\left(s, y_{s}^{(n)}+\bar{\phi}_{s}\right) d s \\
& \left.\left.-\sum_{k=1}^{m} S_{\alpha}\left(b-t_{k}\right) I_{k}\left(y^{(n)}\left(t_{k}^{-}\right)+\bar{\phi}\left(t_{k}^{-}\right)\right)\right](\eta) d \eta\right\} \\
& -\left\{\bar{\rho}_{*}(t)-\sum_{0<t_{k}<t} S_{\alpha}\left(t-t_{k}\right) I_{k}\left(y^{(*)}\left(t_{k}^{-}\right)+\bar{\phi}\left(t_{k}^{-}\right)\right)\right. \\
& -\int_{0}^{t}(t-\eta)^{\alpha-1} T_{\alpha}(t-\eta) B W^{-1}\left[x_{1}-S_{\alpha}(b)[\phi(0)-g(0, \phi)]-g\left(b, y_{b}^{(*)}+\bar{\phi}_{b}\right)\right.
\end{aligned}
$$




$$
\begin{aligned}
& -\int_{0}^{b}(b-s)^{\alpha-1} A T_{\alpha}(b-s) g\left(s, y_{s}^{(*)}+\bar{\phi}_{s}\right) d s \\
& \left.\left.-\sum_{k=1}^{m} S_{\alpha}\left(b-t_{k}\right) I_{k}\left(y^{(*)}\left(t_{k}^{-}\right)+\bar{\phi}\left(t_{k}^{-}\right)\right)\right](\eta) d \eta\right\} \|_{b} \\
& \longrightarrow 0, \quad \text { as } n \longrightarrow \infty .
\end{aligned}
$$

Consider the linear continuous operator

$$
\begin{aligned}
& \Gamma: L^{1}(J, X) \longrightarrow C(J, X) \\
& f \longrightarrow \Gamma(f)(t)=\int_{0}^{t}(t-s)^{\alpha-1} T_{\alpha}(t-s)\left[f(s)+B W^{-1} \int_{0}^{b}(b-\tau) T_{\alpha}(b-\tau) f(\tau) d \tau\right](s) d s .
\end{aligned}
$$

From Lemma 3.1, it follows that $T \circ S_{F}$ is a closed graph operator. Moreover, we have

$$
\begin{aligned}
\bar{\rho}_{n}(t) & -\sum_{0<t_{k}<t} S_{\alpha}\left(t-t_{k}\right) I_{k}\left(y^{(n)}\left(t_{k}^{-}\right)+\bar{\phi}\left(t_{k}^{-}\right)\right) \\
& -\int_{0}^{t}(t-\eta)^{\alpha-1} T_{\alpha}(t-\eta) B W^{-1}\left[x_{1}-S_{\alpha}(b)[\phi(0)-g(0, \phi)]-g\left(b, y_{b}^{(n)}+\bar{\phi}_{b}\right)\right. \\
& -\int_{0}^{b}(b-s)^{\alpha-1} A T_{\alpha}(b-s) g\left(s, y_{s}^{(n)}+\bar{\phi}_{s}\right) d s \\
& \left.-\sum_{k=1}^{m} S_{\alpha}\left(b-t_{k}\right) I_{k}\left(y^{(n)}\left(t_{k}^{-}\right)+\bar{\phi}\left(t_{k}^{-}\right)\right)\right](\eta) d \eta \in \Gamma\left(S_{\left.F, y^{(n)}\right)} .\right.
\end{aligned}
$$

Since $y^{(n)} \longrightarrow y^{*}$, it follows from Lemma 3.2 that

$$
\begin{aligned}
\bar{\rho}_{*}(t) & -\sum_{0<t_{k}<t} S_{\alpha}\left(t-t_{k}\right) I_{k}\left(y^{(*)}\left(t_{k}^{-}\right)+\bar{\phi}\left(t_{k}^{-}\right)\right) \\
& -\int_{0}^{t}(t-\eta)^{\alpha-1} T_{\alpha}(t-\eta) B W^{-1}\left[x_{1}-S_{\alpha}(b)[\phi(0)-g(0, \phi)]-g\left(b, y_{b}^{(*)}+\bar{\phi}_{b}\right)\right. \\
& -\int_{0}^{b}(b-s)^{\alpha-1} A T_{\alpha}(b-s) g\left(s, y_{s}^{(*)}+\bar{\phi}_{s}\right) d s \\
& \left.-\sum_{k=1}^{m} S_{\alpha}\left(b-t_{k}\right) I_{k}\left(y^{(*)}\left(t_{k}^{-}\right)+\bar{\phi}\left(t_{k}^{-}\right)\right)\right](\eta) d \eta \\
= & \int_{0}^{t}(t-s)^{\alpha-1} T_{\alpha}(t-s)\left[f_{*}(s)+B W^{-1} \int_{0}^{b}(b-\tau) T_{\alpha}(b-\tau) f_{*}(\tau) d \tau\right](s) d s
\end{aligned}
$$

for some $f_{*} \in S_{F, y^{*}}$. Hence $\Phi_{2}$ is a completely continuous multivalued map, u.s.c. with convex closed values.

Step 7. The operator inclusions $y \in \Phi_{1} y+\Phi_{2} y=\Phi y$ has a solution in $\mathfrak{B}_{b}^{0}$. 
Let $y$ be a possible solution of $y \in \lambda \Phi(y)$ for some $\lambda \in(0,1)$. Then there exists $f \in S_{F, y}$ such that, for $t \in J$, we have

$$
\begin{aligned}
& y(t)=\lambda\left[-S_{\alpha}(t) g(0, \phi)+g\left(t, y_{t}+\bar{\phi}_{t}\right)+\int_{0}^{t}(t-s)^{\alpha-1} A T_{\alpha}(t-s) g\left(s, y_{s}+\bar{\phi}_{s}\right) d s\right. \\
& +\int_{0}^{t}(t-s)^{\alpha-1} T_{\alpha}(t-s) f(s) d s+\sum_{0<t_{k}<t} S_{\alpha}\left(t-t_{k}\right) I_{k}\left(y\left(t_{k}^{-}\right)+\bar{\phi}\left(t_{k}^{-}\right)\right) \\
& +\int_{0}^{t}(t-\eta)^{\alpha-1} T_{\alpha}(t-\eta) B W^{-1}\left[x_{1}-S_{\alpha}(b)[\phi(0)-g(0, \phi)]-g\left(b, y_{b}+\bar{\phi}_{b}\right)\right. \\
& -\int_{0}^{b}(b-s)^{\alpha-1} A T_{\alpha}(b-s) g\left(s, y_{s}+\bar{\phi}_{s}\right) d s-\int_{0}^{b}(b-s)^{\alpha-1} T_{\alpha}(b-s) f(s) d s \\
& \left.\left.-\sum_{k=1}^{m} S_{\alpha}\left(b-t_{k}\right) I_{k}\left(y\left(t_{k}^{-}\right)+\bar{\phi}\left(t_{k}^{-}\right)\right)\right](\eta) d \eta\right], \quad t \in J, \\
& |y(t)| \leq M\left[\left\|(-A)^{-\beta}\right\|\left(c_{1}\|\phi\|_{\mathfrak{B}_{h}}+c_{2}\right)\right]+\left\|(-A)^{-\beta}\right\|\left(c_{1}\left\|y_{t}+\bar{\phi}_{t}\right\|_{\mathfrak{B}_{h}}+c_{2}\right) \\
& +\int_{0}^{t} \frac{\alpha c_{1-\beta} \Gamma(1+\beta)}{(t-s)^{\alpha(1-\beta)} \Gamma(1+\alpha \beta)} \cdot(t-s)^{\alpha-1}\left(c_{1}\left\|y_{t}+\bar{\phi}_{t}\right\|_{\mathfrak{B}_{h}}+c_{2}\right) d s \\
& +\int_{0}^{t}(t-s)^{\alpha-1} \frac{\alpha M}{\Gamma(1+\alpha)} p(s) \psi\left(\left\|y_{t}+\bar{\phi}_{t}\right\|_{\mathfrak{B}_{h}}\right) d s+M \sum_{k=1}^{m} d_{k} \\
& +\frac{\alpha M M_{2} M_{3}}{\Gamma(1+\alpha)} \int_{0}^{t}(t-\eta)^{\alpha-1} \mid x_{1}-S_{\alpha}(b)[\phi(0)-g(0, \phi)]-g\left(b, y_{b}+\bar{\phi}_{b}\right) \\
& -\int_{0}^{b}(b-s)^{\alpha-1} A T_{\alpha}(b-s) g\left(s, y_{s}+\bar{\phi}_{s}\right) d s \\
& -\int_{0}^{b}(b-s)^{\alpha-1} T_{\alpha}(b-s) f(s) d s \\
& -\sum_{k=1}^{m} S_{\alpha}\left(b-t_{k}\right) I_{k}\left(y\left(t_{k}^{-}\right)+\bar{\phi}\left(t_{k}^{-}\right)\right) \mid(\eta) d \eta \\
& \leq M\left[\left\|(-A)^{-\beta}\right\|\left(c_{1}\|\phi\|_{\mathfrak{B}_{h}}+c_{2}\right)\right]+\left\|(-A)^{-\beta}\right\|\left(c_{1}\left\|y_{t}+\bar{\phi}_{t}\right\|_{\mathfrak{B}_{h}}+c_{2}\right) \\
& +\int_{0}^{t} \frac{\alpha c_{1-\beta} \Gamma(1+\beta)}{\Gamma(1+\alpha \beta)} \cdot(t-s)^{\alpha \beta-1}\left(c_{1}\left\|y_{t}+\bar{\phi}_{t}\right\|_{\mathfrak{B}_{h}}+c_{2}\right) d s \\
& +\int_{0}^{t}(t-s)^{\alpha-1} \frac{\alpha M}{\Gamma(1+\alpha)} p(s) \psi\left(\left\|y_{s}+\bar{\phi}_{s}\right\|_{\mathfrak{B}_{h}}\right) d s+M \sum_{k=1}^{m} d_{k} \\
& +\frac{\alpha M M_{2} M_{3}}{\Gamma(1+\alpha)} \int_{0}^{t}(t-\eta)^{\alpha-1}\left\{\left|x_{1}\right|+M\left[\phi(0)+\left\|(-A)^{-\beta}\right\|\left(c_{1}\|\phi\|_{\mathfrak{B}_{h}}+c_{2}\right)\right]\right. \\
& +\left\|(-A)^{-\beta}\right\|\left(c_{1}\left\|y_{b}+\bar{\phi}_{b}\right\|_{\mathfrak{B}_{h}}+c_{2}\right) \\
& +\int_{0}^{b} \frac{\alpha c_{1-\beta} \Gamma(1+\beta)}{\Gamma(1+\alpha \beta)} \cdot(b-s)^{\alpha \beta-1}\left(c_{1}\left\|y_{s}+\bar{\phi}_{s}\right\|_{\mathfrak{B}_{h}}+c_{2}\right) d s \\
& \left.+\int_{0}^{b}(b-s)^{\alpha-1} \frac{\alpha M}{\Gamma(1+\alpha)} p(s) \psi\left(\left\|y_{s}+\bar{\phi}_{s}\right\|_{\mathfrak{B}_{h}}\right) d s+M \sum_{k=1}^{m} d_{k}\right\}(\eta) d \eta .
\end{aligned}
$$


Since $\left\|y_{t}+\bar{\phi}_{t}\right\|_{\mathfrak{B}_{h}} \leq l \sup _{s \in[0, t]}|y(s)|+\|\phi\|_{\mathfrak{B}_{h}}+l M|\phi(0)|$,

$$
\begin{aligned}
\|y\|_{b}= & \sup _{t \in[0, b]}|y(t)| \\
\leq & M\left[\left\|(-A)^{-\beta}\right\|\left(c_{1}\|\phi\|_{\mathfrak{B}_{h}}+c_{2}\right)\right] \\
& +\left\|(-A)^{-\beta}\right\|\left(c_{1} l \sup _{t \in[0, b]}|y(t)|+c_{1}\|\phi\|_{\mathfrak{B}_{h}}+c_{1} l M|\phi(0)|+c_{2}\right) \\
& +\frac{\alpha c_{1-\beta} \Gamma(1+\beta)}{\Gamma(1+\alpha \beta)} \cdot \frac{b^{\alpha \beta}}{\alpha \beta}\left(c_{1} l \sup _{t \in[0, b]}|y(t)|+c_{1}\|\phi\|_{\mathfrak{B}_{h}}+c_{1} l M|\phi(0)|+c_{2}\right) \\
& +\sup _{s \in J} p(s) \frac{b^{\alpha} M}{\Gamma(1+\alpha)} \psi\left(l \sup _{t \in[0, b]}|y(t)|+\|\phi\|_{\mathfrak{B}_{h}}+l M|\phi(0)|\right)+M \sum_{k=1}^{m} d_{k} \\
& +\frac{\alpha M M_{2} M_{3}}{\Gamma(1+\alpha)} \int_{0}^{t}(t-\eta)^{\alpha-1}\left\{\left|x_{1}\right|+M\left[\phi(0)+\left\|(-A)^{-\beta}\right\|\left(c_{1}\|\phi\|_{\mathfrak{B}_{h}}+c_{2}\right)\right]\right. \\
& +\left\|(-A)^{-\beta}\right\|\left(c_{1} l \sup _{t \in[0, b]}|y(t)|+c_{1}\|\phi\|_{\mathfrak{B}_{h}}+c_{1} l M|\phi(0)|+c_{2}\right) \\
& +\left(c_{1} l \sup _{t \in[0, b]}|y(t)|+c_{1}\|\phi\|_{\mathfrak{B}_{h}}+c_{1} l M|\phi(0)|+c_{2}\right) \frac{c_{1-\beta} \Gamma(1+\beta)}{\Gamma(1+\alpha \beta)} \cdot \frac{b^{\alpha \beta}}{\beta} \\
& \left.+\sup _{s \in J} p(s) \frac{b^{\alpha} M}{\Gamma(1+\alpha)} \psi\left(l \sup _{t \in[0, b]}|y(t)|+\|\phi\|_{\mathfrak{B}_{h}}+l M|\phi(0)|\right)+M \sum_{k=1}^{m} d_{k}\right\}(\eta) d \eta \\
\leq & F_{1}+F_{2}\|y\|_{b}+F_{3} \psi\left(l\|y\|_{b}+\|\phi\|_{\mathfrak{B}_{h}}+l M|\phi(0)|\right),
\end{aligned}
$$

where $F_{1}, F_{2}, F_{3}$ are defined in $\left(H_{6}\right)$.

So $\|y\|_{b} \leq F_{1}+F_{2}\|y\|_{b}+F_{3} \psi\left(l\|y\|_{b}+\|\phi\|_{\mathfrak{B}_{h}}+l M|\phi(0)|\right)$, that is,

$$
\frac{\|y\|_{b}}{F_{1}+F_{2}\|y\|_{b}+F_{3} \psi\left(l\|y\|_{b}+\|\phi\|_{\mathfrak{B}_{h}}+l M|\phi(0)|\right)} \leq 1
$$

Then by $\left(H_{6}\right)$ there exists $r$ such that $\|y\|_{b} \neq r$. Hence, it follows from Theorem 2.2 that the operator $\Phi$ has a fixed point $y^{*} \in \mathfrak{B}_{b}^{0}$. Let $x(t)=y^{*}(t)+\bar{\phi}(t), t \in(-\infty, b]$. Then $x$ is a fixed point of the operator $\mathfrak{L}$ which is a mild solution of problem (1.1); then system (1.1) is controllable on $J$.

\section{Conclusion}

In this paper, we have investigated the controllability of fractional impulsive neutral functional differential inclusions in Banach spaces. Based on a fixed point theorem, sufficient conditions for the controllability of the fractional impulsive neutral functional differential inclusions have been derived.

Competing interests

The author declares that he has no competing interests.

\section{Acknowledgements}

This work was supported by the grant of Chongqing municipal educational commission (No: KJ120609), Natural Science Foundation Project of CSTC, 2014jcyjA00015, the PhD Foundation of Chongqing Normal University under Grant No. 09XLB007. 


\section{References}

1. Kilbas, AA, Srivastava, HM, Trujillo, JJ: Theory and Applications of Fractional Differential Equations. North-Holland Mathematics Studies, vol. 204. Elsevier, Amsterdam (2006)

2. Lakshmikantham, V, Leela, S, Devi, JV: Theory of Fractional Dynamic Systems. Cambridge Scientific Publishers, Cambridge (2009)

3. Miller, KS, Ross, B: An Introduction to the Fractional Calculus and Differential Equations. Wiley, New York (1993)

4. Podlubny, I: Fractional Differential Equations. Academic Press, San Diego (1999)

5. Agarwal, RP, Benchohra, M, Hamani, S: A survey on existence results for boundary value problems of nonlinear fractional differential equations and inclusions. Acta Appl. Math. 109, 973-1033 (2010)

6. Bainov, DD, Simeonov, PS: Impulsive Differential Equations: Periodic Solutions and Applications. Longman, New York (1993)

7. Lakshmikantham, V, Bainov, DD, Simeonov, PS: Theory of Impulsive Differential Equations. World Scientific, Singapore (1989)

8. Yang, T: Impulsive Control Theory. Springer, Berlin (2001)

9. Wang, J, Zhou, Y: Existence and controllability results for fractional semilinear differential inclusions. Nonlinear Anal., Real World Appl. 12, 3642-3653 (2011)

10. Wang, J, Zhou, Y: Complete controllability of fractional evolution systems. Commun. Nonlinear Sci. Numer. Simul. 17, 4346-4355 (2012)

11. Wang, J, Fan, Z, Zhou, Y: Nonlocal controllability of semilinear dynamic systems with fractional derivative in Banach spaces. J. Optim. Theory Appl. 154, 292-302 (2012)

12. Feckan, M, Wang, J, Zhou, Y: Controllability of fractional functional evolution equations of Sobolev type via characteristic solution operators. J. Optim. Theory Appl. 156, 79-95 (2013)

13. Wang, J, Zhou, Y: Analysis of nonlinear fractional control systems in Banach spaces. Nonlinear Anal. TMA 74 5929-5942 (2011)

14. Wang, J, Zhou, Y, Wei, W: Optimal feedback control for semilinear fractional evolution equations in Banach spaces. Syst. Control Lett. 61, 472-476 (2012)

15. Wang, J, Feckan, M, Zhou, Y: Relaxed controls for nonlinear fractional impulsive evolution equations. J. Optim. Theory Appl. 156, 13-32 (2013)

16. Wang, J, Feckan, M, Zhou, Y: Controllability of Sobolev type fractional evolution equations. Dyn. Partial Differ. Equ. 11, 71-87 (2014)

17. Chang, Y-K, Anguraj, A, Mallika Arjunan, M: Controllability of impulsive neutral functional differential inclusions with infinite delay in Banach space. Chaos Solitons Fractals 39, 1864-1876 (2009)

18. Sakthivel, R, Mahmudov, NI, Nieto, JJ: Controllability for a class of fractional-order neutral evolution control systems. Appl. Math. Comput. 218, 10334-10340 (2012)

19. Yan, Z: Approximate controllability of partial neutral functional differential systems of fractional order with state-dependent delay. Int. J. Control 85, 1051-1062 (2012)

20. Sakthivel, R, Suganya, S, Anthoni, SM: Approximate controllability of fractional stochastic evolution equations. Comput. Math. Appl. 63, 660-668 (2012)

21. Sakthivel, R, Ren, Y: Complete controllability of stochastic evolution equations with jumps. Rep. Math. Phys. 68, 163-174 (2011)

22. Sakthivel, R, Ren, Y, Mahmudov, NI: On the approximate controllability of semilinear fractional differential systems. Comput. Math. Appl. 62, 1451-1459 (2011)

23. Sakthivel, R, Mahmudov, NI, Ren, Y: Approximate controllability of the nonlinear third-order dispersion equation. Appl. Math. Comput. 217, 8507-8511 (2011)

24. Benchohra, M, Gorniewicz, L, Ntouyas, SK: Controllability on infinite time horizon for first and second order functional differential inclusions in a Banach spaces. Discuss. Math., Differ. Incl. Control Optim. 21, 261-282 (2001)

25. Park, JY, Kwun, YC, Lee, HJ: Controllability of second order neutral functional differential inclusions in a Banach spaces. J. Math. Anal. Appl. 285, 37-49 (2003)

26. Liu, B: Controllability of neutral functional differential and integrodifferential inclusions with infinite delay. J. Optim. Theory Appl. 123, 573-593 (2004)

27. Liu, B: Controllability of impulsive neutral functional differential inclusions with infinite delay. Nonlinear Anal. 60 1533-1552 (2005)

28. Balasubramaniam, P, Ntouyas, SK: Controllability for neutral stochastic functional differential inclusions with infinite delay in abstract space. J. Math. Anal. Appl. 324, 161-176 (2006)

29. Dhage, BC: Multivalued mapping and fixed point I. Nonlinear Funct. Anal. Appl. 10, 359-378 (2005)

30. Wang, J, Zhou, Y: A class of fractional evolution equations and optimal controls. Nonlinear Anal. 12, $262-272$ (2011)

31. Wu, J: Theory and Applications of Partial Functional Differential Equations. Springer, New York (1996)

32. Deimling, K: Multivalued Differential Equations. de Gruyter, Berlin (1992)

33. Hu, S, Papageorgiou, N: Handbook of Multivalued Analysis, Theory, vol. 1. Kluwer Academic, Dordrecht (1997)

34. Pazy, A: Semigroups of Linear Operators and Applications to Partial Differential Equations. Springer, New York (1983)

35. Lasota, A, Optal, Z: Application of the Kakutani-Ky-Fan theorem in the theory of ordinary differential equations or noncompact acyclic-valued map. Bull. Acad. Pol. Sci., Sér. Sci. Math. Astron. Phys. 13, 781-786 (1965)

10.1186/1687-1847-2014-234

Cite this article as: Li: Controllability of nonlinear neutral fractional impulsive differential inclusions in Banach space. Advances in Difference Equations 2014, 2014:234 